\title{
Effect of Knee Extensors Strengthening Versus Hip Posterolateral Musculature Strengthening with Ankle Dorsi Flexors in Patellofemoral Pain
}

\author{
Shweta Suryvanshi ${ }^{1 *}$ and Nikhil Kumar Rana ${ }^{2}$ \\ ${ }^{1}$ In Charge, Principal, Knowledge Institute of Physiotherapy, Anand, India \\ ${ }^{2}$ Principal, Goutham College of Physiotherapy, Bangalore, India \\ *Corresponding Author: Shweta Suryvanshi, In Charge, Principal, Knowledge \\ Institute of Physiotherapy, Anand, India.
}

DOI: 10.31080/ASOR.2022.05.0429
Received: March 23, 2021

Published: February 21, 2022

(C) All rights are reserved by Shweta

Suryvanshi and Nikhil Kumar Rana.

\section{Abstract}

Background: Patellofemoral Pain Syndrome (PFPS) is an umbrella term used for pain arising from the patellofemoral joint itself, or adjacent soft tissues. The knee consists of two major joints tibiofemoral and patellofemoral commonly affected as patellofemoral joint pain with lower extremity dysfunction that physiotherapists and sports specialist rehabilitate. Mostly the anterior knee pain was caused by joint stress at the patella. This may be occured by chondromalacia patella or malalignment of the femur during recreational activities. Rehabilitation mainly focus on relieving pain maintaining the momentum positioning of the patella on femur and may incorporate quadriceps reinforcement, coxa and back extensor muscle strength training knee flexors and stretching exercise of iliotibial band, patellar glide, taping on patella, external aids for lower base, and pelvis manual therapy intervention.

Objective: Main aim: 1. To analyze the outcome of quadriceps muscle build up versus abductors and extensors group of muscle with dorsiflexors muscle of ankle joint training on betterment of genus activity and having ache in runner's knee pain patient. Another aim: 2. To decide the potency of genus quadriceps muscle reinforce training on measure of functional activity and having stress pain for subjects with anterior knee pain condition.

Materials and Methods: The subjects selected from Random sampling method from variant hospitals in Bengaluru and protocol will be executed in Goutham physiotherapy clinic and rehabilitation center were screened and 50 subjects were divided in two groups i.e., Group A- In this group ankle dorsiflexors with quadriceps muscle strengthening, Group B - strengthening of glutei and hip abductors muscles with ankle dorsiflexors. The explanation of the study was done on the footing analyzing pre-test and post-test evaluation of Pain Gait analysis, Kujala patellofemoral pain syndrome questionnaire. Quadriceps Angle.

Results: Comparison of mean difference of Q - Angle between Group A and Group B. In this when the mean value of Group A (17.2) compared with mean value of Group B (14.52), the mean difference is (2.68), the t' value is (5.154), and ' $p$ ' value is 0.0013 which is highly significant.

Conclusion: There were significantly decrease in pain with hip posterolateral musculature strengthening exercise with ankle dorsi flexion than the Knee extensors strengthening. Hip Posterolateral Musculature Strengthening exercise with ankle dorsi flexion were decreases in VAS and improving in Q - Angle towards the normal angle. Knee Extensors Strengthening exercises have not such an important intervention for anterior knee pain condition. Patellofemoral Pain Syndrome relieves with Hip Posterolateral Musculature strengthening exercises with ankle dorsi flexion.

Keywords: Patellofemoral Pain Syndrome (PFPS); Knee; Ankle

Citation: Shweta Suryvanshi and Nikhil Kumar Rana. "Effect of Knee Extensors Strengthening Versus Hip Posterolateral Musculature Strengthening with Ankle Dorsi Flexors in Patellofemoral Pain". Acta Scientific Orthopaedics 5.3 (2021): 49-56. 


\section{Introduction}

Runner's knee pain syndrome (PFPS) is the therapeutic meaning for pain in the front of the knee, usually under the patella. It is also known as jumper's knee is a basic lower extremity condition that health care workers and sports specialist rehabilitate. Symptoms is commonly increase with climbing, full squat, lunges, speedy dynamic activities and sitting for a prolonged time. There are multifactorial causes mainly occurred by overused knee joint with structural damage in cartilage. Mostly the anterior knee discomfort was caused by joint stress at the patella. This may be occurred by chondromalacia patella or malalignment of the femur during recreational activities. Rehabilitation focus on rest on knee and maintaining the momentum alignment of the patellofemoral angle it may involve genus quadriceps facilitation, coxa and muscle strength training of back extensors, knee flexors and stretching exercise of iliotibial band, patellar glide, kinetic taping, external aids for lower base, and pelvis manual therapy intervention [1] annual prevalence rate for anterior knee ache was $22.7 \%$. women were 2.23 counts $(95 \% \mathrm{CI}: 1.19,4.20)$ commonly occur chondromalacia patella in contrast with males. That the prevalence of PFPS not significantly. different between both the sexes at the time of admission to the US Naval Academy. This data along with other studies suggests that females are more affected than males by a rapid increase in physical activity level, which in turn leads to a higher incidence of PFPS [2] one of the main sticking words was found on this content, firstly, quadriceps inhibition is a major factor for initiating pain around the anterior aspect of knee, knee extensors strengthening is essential phase of rehabilitation for patients. Secondly, limited ankle movement, it may create biomechanical misalignment it required keen observation by therapist because there is a chances of angular deviation with an anterior translation of weight during close kinematic activities. an athlete present history of patellofemoral pain present with decreased ankle extension movement, it was suggested that distal mechanical factors should be clinically addressed when a patient present with late excessive rear-foot eversion during walking. This has result in patellofemoral joint loading because prominent work done by everting talus in the same timing and combined motion within the affected lower extremity. As eversion reaches its maximum at mid-stance both tibia medial rotation and hip adduction are going to enhance patellofemoral joint stress [3].
Major hip muscles to strengthen include:

- $\quad$ Hip abductors: Gluteus medius

- Hip external rotators: Gluteus maximus

- Hip extensors: Gluteus maximus.

Task oriented skillful activities of the lateral side hip musculature have been coordinated into physiotherapy rehabilitation protocol for managing pain, disability, and function in patients with patellofemoral pain [4]. lower extremity injuries contribute limited hip extension motion and because of that reduced speed, coxa shift angle and step length. The major movers are the quadriceps, knee flexors, and glutei for hip extension in one leg standing. The hip and knee rate of change of velocity induced by four muscles during stance. It manifests that the glutei muscles, vastus intermedialis, lateralis and medialis helps in early to mid-stance phase to initiate extension, and in swing to weight bearing phase soleus muscle speed up the energy. All the antigravity muscles stabilize the hip and knee extension during the weight bearing phase of locomotion. Coxa lateral rotation and abductor group of muscles help to maintain leg alignment by isotonic contraction femoral medial rotation and influencing hip adduction stabilizing pelvis during close kinematic activities, inhibition of these muscles may enhance medial femoral rotation, genu valgus, Trendelenburg gait. the abnormalities of these osseous or soft tissue structures may predispose patellofemoral joint compressive force can result in patellofemoral pain, Tightness of these lateral structures may cause a pull on the patella in the Progressing flexion to $60^{\circ}$, contact pressure increases and moves from distal to proximal which subsequently an increased Quadriceps angle, alter tracking of the patella, increase compressive forces on the patellofemoral joint, and ultimately lead to knee pain [5]. Tighten lower extremity ligament, muscle tendons distract the patella laterally and enhance creep effect over the joint prolong time. The primary aim of runner's syndrome is to efficiently achieve the high functional performance [6]. literature suggest knee extensors and hip posterolateral musculature strengthening improves patellofemoral pain syndrome. Very few researches have studied the effect of ankle dorsiflexors muscle strengthening on PFPS. The purpose of research is to measure the efficiency of genus quadriceps, coxa abductors and extensors group of muscles with antigravity muscle of foot strength in patellar pain condition because of that they can improve their functional activities of rou- 
tine life. Along with this correction in acute knee joint angle occur in patient suffering with malalignment in knee joint, so subject. we deliberate Whether there is variation in the usefulness of knee extensor strengthening versus hip posterolateral musculature strengthening with ankle dorsiflexors on functional capabilities and pain for subjects with patellofemoral pain syndrome?

\section{Methodology}

- $\quad$ Research plan: Equivalent experimental study design Pre to post-test two group.

- Sample size: 50 subjects

- Selection method: Random sampling method.

- Data source: Variant hospitals from Bangalore patients were selected and rehabilitation protocol will be plan out in Goutham physiotherapy clinic and other physiotherapy center.

- Study duration: 8 months.

Sample selection

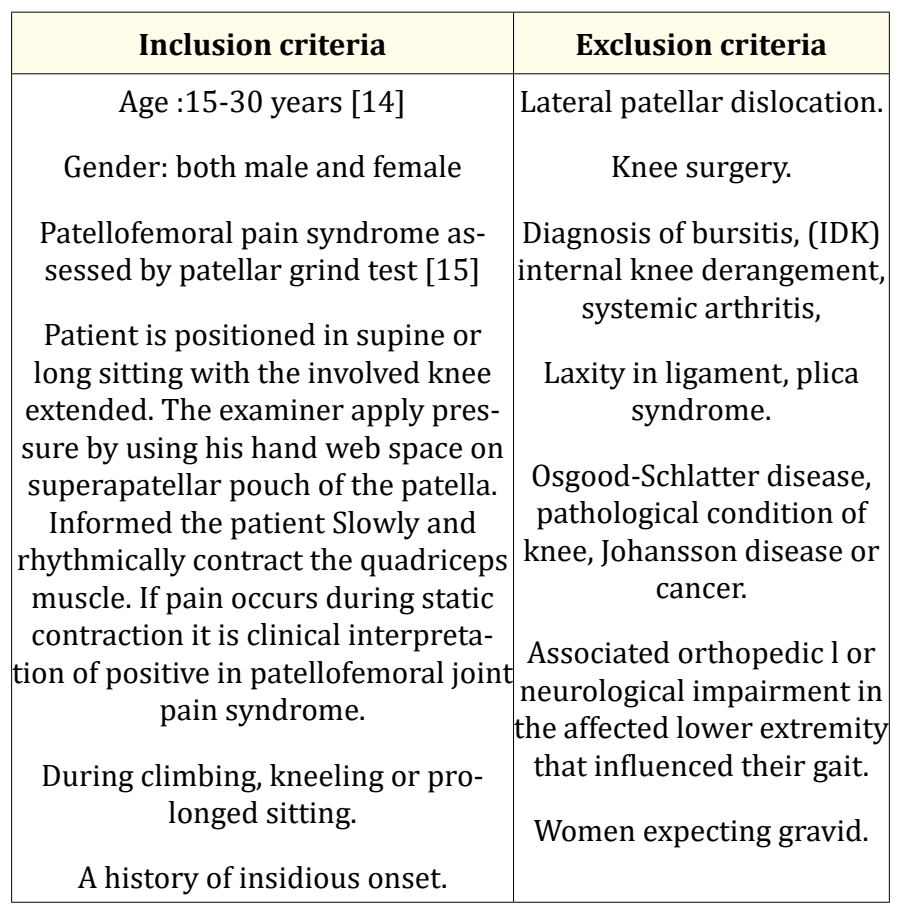

Table a
Variables

Predictable variables

Coxa Posterolateral musculature strengthening, quadriceps strengthening exercises with ankle dorsiflexors, patellofemoral pain syndrome.

Response variables

Functional genus joint and pain.

Materials used

- $\quad$ Plinth

- $\quad$ Ball pen

- $\quad$ Time marker

- Knee extensor table.

- $\quad$ Sand bag.

- Stool

- Paper.

Rehabilitation to be supervise on the subjects

On the basis of selection criteria, 50 subject divided into two group 1-25 subjects and group 2-25 subjects.

Consent with full knowledge will be taken.

- Group A - In this group extensors of ankle with knee extensors muscle strengthening,

- Group B - Hip abductors and extensors muscles with ankle dorsiflexors strength training.

Four weeks' rehabilitation session was given. It is depending on the standard protocol of the progressive resistance exercise technique [13].

Outcome measures will be reassessed and pre and post result are compared, At the end of physical therapy program.

\section{Procedure}

The resistance during training was calculated seventeen percentage of the estimated 1-repetition maximum.

It is known as the maximum load with which 1 repetition of the exercise could be completed without pain. 
The resistance training standards were basis for the use of elastic resistance like Thera band and weight cuff and sand bags are exercise equipment were each patient was able to perform 10 repetitions (10 RM) of the exercise. Reevaluation of resistance during the first treatment session and checked weekly to adjust the maximum load for all strengthening exercises as needed.

The patients were informed to maintain their normal activities of daily life without performing a home exercise after the 2-week of treatment protocol.

Group 1 knee extensor group: According to the daily adjustable progressive resistance exercise regimen training will be conducted on subject positioned in supine lying and high sitting with mechanical weights.

Group 2 posterolateral structures hip strengthening: Three groups of muscle, firstly abductors group strengthening will be done with subjects in side lying position, and secondly lateral rotators training will be done with subject positioned in supine lying with legs out of the couch and third is hip extensor strengthening in prone lying with mechanical weights will be done as per the daily adjustable progressive resistance exercise regimen criteria.

The daily adjustable progressive resistance exercise regimen

Resistance training technique is more standardized interventional and conditioning programs it takes into consideration the distinguish values at which individual's progress. 6 RM working weight is the basis of the procedure.

The working weight of the next exercise session will be determining by the maximum number of repetitions possible using the working wait in a set no 3 of the regimen [20].

Ankle dorsiflexors strengthening

- Heel raise: Perform 3 sets of 10 repetition of ankle strengthening exercise start with standing at a bench or chair for balance. Maintaining feet shoulder width apart and facing ahead. move up gradually onto your toes, Heels should be raised as far as possible and comfortable without pain.

- Single leg calf raise: Perform 3 sets of 10 repetitions, standing on one leg at a table Or chair for balance. Maintaining

\begin{tabular}{|c|}
\hline DAPRE Technique \\
\hline Sets Repetitions Amount of Resistance \\
\hline $1050 \% 6 \mathrm{RM}$ \\
$675 \% 6 \mathrm{RM}$ \\
Maximum possible 100\% $6 \mathrm{RM}$ \\
Maximum possible 100\% adjusted working weight \\
\hline
\end{tabular}

Table b

your foot facing forwards, without having any discomfort try to go up gradually onto your toes with inclined on heel.

- $\quad$ Single leg calf raises over a step with weight: building up the power of small muscle of foot exercise begins with Sound leg standing and lifting a light dumbbell with your heel dropped below the level of a step. raising your heel as far as possible and comfortable without pain Slowly move up onto your toes. The exercise can be improved by gradually increasing the weight once one is mastered in it.

Pre and post test evaluation

- $\quad$ Pain

- Gait analysis.

Outcome measures

- $\quad$ Quadriceps Angle (Q Angle).

- Kujala patellofemoral pain syndrome questionnaire.

\section{Statistical Methods}

Descriptive statistical analysis has been carried out in the present study. Out Come measurements of Visual Analog Scale and Q- Angle was measured in group - A and with group - B. Outcome score for the subjects studied and presented as mean SD. Significance is assessed at $5 \%$ level of significance with $p$ value was set at 0.10 (and doubled for 2 tailed Hypothesis) less than this is considered as statistically significant difference

\section{Statistical tests}

SPSS software for window (version16) is used for Statistical analysis and $\mathrm{p}$ value will be set as 0.10 (2-tailed hypothesis). Baseline demographic data analyzed by descriptive statistics and chi square test. 
Independent $\mathrm{t}$ test and ranked sum test (nonparametric test) will be applied to evaluate the significance of parameters Values.

The significance of parameters between the groups will be find by Independent $t$ test and Wilcoxon signed ranked test.

\section{Results}

The table and graphs illustrate outcome measurement analysis between group-A and Group-B.

- $\quad$ Group - A - Subjects with foot extensors with quadriceps muscle strengthening

- $\quad$ Group - B - Subjects with strengthening of hip posterolateral muscle strengthening with ankle dorsi flexion.

On the basis of proportional Sampling Method all the subjects will be included.
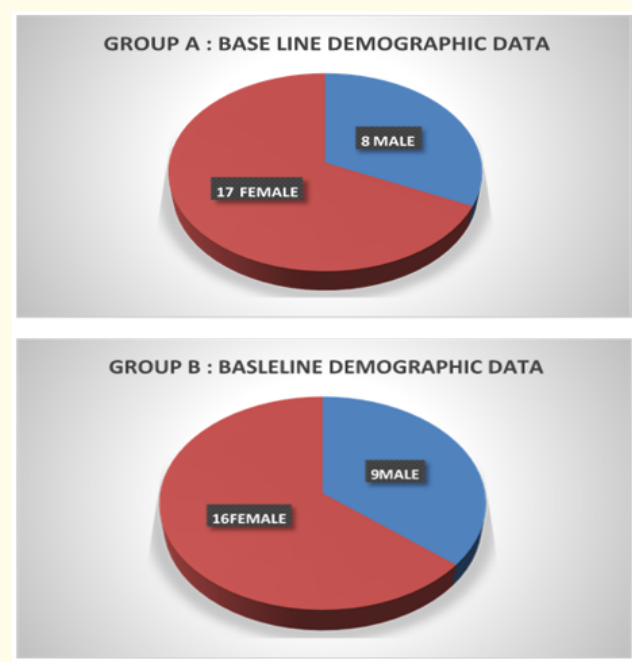

Figure a

\begin{tabular}{|c|c|c|}
\hline Subject & Group a & Group b \\
\hline Male & 8 & 9 \\
\hline Female & 17 & 16 \\
\hline Total & 25 & 25 \\
\hline
\end{tabular}

Table 1: Male and female base_line data.

\begin{tabular}{|c|c|c|c|c|c|}
\hline & & \multicolumn{2}{|c|}{ Q - Angle } & & \\
\cline { 1 - 5 } & Mean & Mean. Diff & S.D & t - Value & p - Value \\
\cline { 1 - 2 } Group A & 17.2 & 2.68 & 2.236 & 5.154 & 0.0013 \\
\cline { 1 - 2 } \cline { 1 - 4 } Group & 14.52 & & 1.327 & & \\
\hline
\end{tabular}

Table 2: Comparison of Post Interventional Value Q - Angle between Group A and Group B.

Comparison of mean difference of Q - Angle between Group A - with ankle dorsa flexion with knee extensors muscle strengthening exercise and Group B - with strengthening of posterolateral hip muscle strengthening with ankle dorsi flexion.

In this when the mean value of Group A (17.2) compared with mean value of Group B (14.52), the mean difference is (2.68), the ' $t$ ' value is (5.154), and 'p' value is 0.0013 which is highly significant.

\begin{tabular}{|c|c|c|c|c|c|}
\hline \multicolumn{7}{|c|}{ Numeric pain rating scale } \\
\hline & Mean & Mean dif. & S.D & t-value & p-value \\
\hline Group A & 4.28 & 3.48 & 1.173 & 12.17 & 0.082 \\
\hline Group B & 0.8 & & 0.8165 & & \\
\hline
\end{tabular}

Table 3: Comparison of Post Interventional Value Between Group A and Group B.

Comparison of mean difference of Visual Analog Scale between Group A - with ankle dorsi flexion with knee extensors muscle strengthening exercise and Group B - with strengthening of posterolateral hip muscle strengthening with ankle dorsi flexion. In this when the mean value of Group A (4.28) compared with mean value of Group B (0.0800), the mean difference is (3.48), the $t$ ' value is (12.17), and 'p' value is (0.082) which is highly significant.

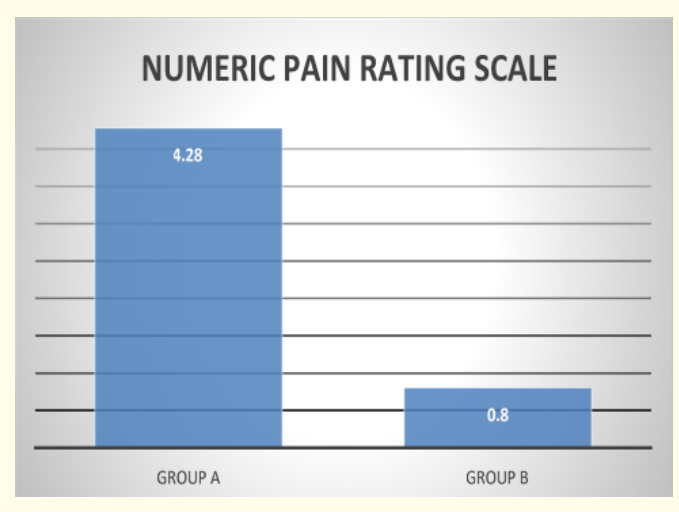

Chart 1: Comparison of Post Interventional Value Between Group A and Group B.

Citation: Shweta Suryvanshi and Nikhil Kumar Rana. "Effect of Knee Extensors Strengthening Versus Hip Posterolateral Musculature Strengthening with Ankle Dorsi Flexors in Patellofemoral Pain". Acta Scientific Orthopaedics 5.3 (2021): 49-56. 


\begin{tabular}{|c|c|}
\hline \multicolumn{2}{|c|}{ Prevalence of patellofemoral pain } \\
\hline Male & 17 \\
\hline Female & 33 \\
\hline
\end{tabular}

Table 4: Total Prevalence of Patellofemoral Pain Syndrome.

This study is a comparative experimental study design between two groups involving the subjects with patellofemoral pain. The subjects for this study were included on the basis of Random Sampling Method.

There was total no of subjects are 50 subjects. This shows that the prevalence of patellofemoral pain syndrome is mainly seen in Female than the men. Due to increased Q - angle in Female.

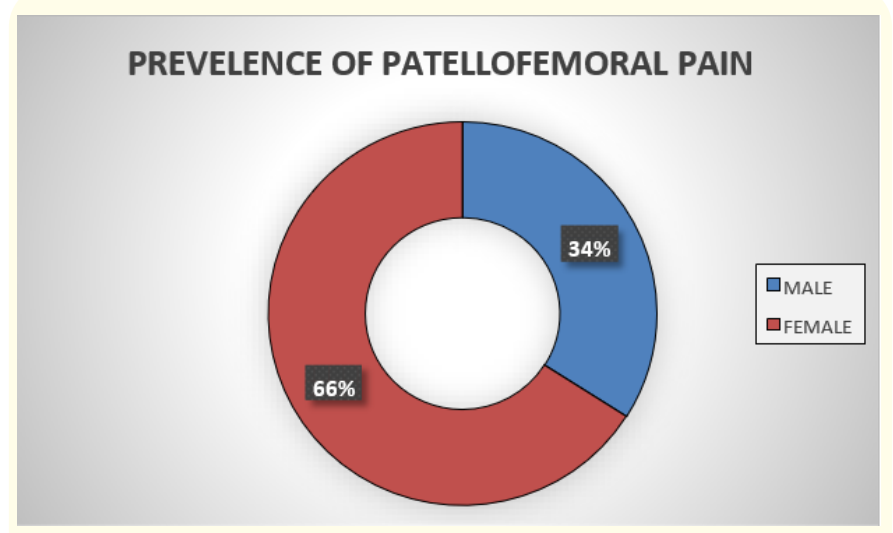

Chart 2: Total Prevalence of Patellofemoral Pain Syndrome.

\section{Discussion}

According to the results obtained from the study titled "Effect of knee extensors strengthening versus hip posterolateral musculature strengthening with ankle dorsi flexors in patellofemoral pain". it was concluded that the Hip Posterolateral Musculature Strengthening with Ankle Dorsi Flexion Exercises is more effective than the Knee Extensor Strengthening Exercises.

The analysis of outcome measurement B/W group A and Group $B$ has been presented in tables and graphs.
- $\quad$ Group - A - Subjects with ankle dorsi flexion with knee extensors muscle strengthening

- $\quad$ Group - B - Subjects with strengthening of posterolateral hip muscle strengthening with ankle dorsi flexion.

Comparison of mean difference of Q - Angle between Group A - with ankle dorsi flexion with knee extensors muscle strengthening exercise and Group B - with strengthening of posterolateral hip muscle strengthening with ankle dorsi flexion.

In this when the mean value of Group A (17.2) compared with mean value of Group B (14.52), the mean difference is (2.68), the ' $t$ ' value is (5.154), and 'p' value is 0.0013 which is highly significant.

So this study shows that the interventional program with strengthening of posterolateral hip muscles strengthening with ankle dorsi flexion is more effective in patellofemoral pain syndrome.

Due to hip posterolateral musculature strengthening exercise $Q$ - Angle will come to its normal range.

Comparison of mean difference of Visual Analog Scale between Group A - with ankle dorsi flexion with knee extensors muscle strengthening exercise and Group B - with strengthening of posterolateral hip muscle strengthening with ankle dorsi flexion. In this when the mean value of Group A (4.28) compared with mean value of Group B (0.0800), the mean difference is (3.48), the $t^{\prime}$ value is (12.17), and ' $p$ ' value is (0.082) which is highly significant.

Due to posterolateral musculature strengthening exercise with ankle dorsi flexion will leads to decreases the weight bearing to medial side of the knee. So that the equal weight bearing comes under the knee joint and the patellofemoral joint will have efficient function while walking. Due to decreased medial side weight bearing pain is reduced by hip posterolateral musculature exercise with ankle dorsi flexion.

This study is a comparative experimental study design between two groups involving the subjects with patellofemoral pain. The subjects for this study were included on the basis of Random Sampling Method. 
There were total number of subjects are 50 subjects. This shows that the prevalence of patellofemoral pain syndrome is mainly seen in Female than the men. Due to increased Q - Angle in Female.

\section{Conclusion}

- $\quad$ There were significantly decrease in pain with hip posterolateral musculature strengthening exercise with ankle dorsi flexion than the Knee extensors strengthening.

- Hip Posterolateral Musculature Strengthening exercise with ankle dorsi flexion were decreases in VAS and improving in Q - Angle towards the normal angle.

- Knee Extensors Strengthening exercises have not comparatively so much an efficient treatment for Patellofemoral Pain Syndrome.

- Patellofemoral Pain Syndrome relieves with Hip Posterolateral Musculature strengthening exercises with ankle dorsi flexion.

\section{Limitations of the Study}

- $\quad$ Sample size is small.

- There EMG Study was not done.

- Electrotherapy or Modalities can be given.

- Intervention should be divided into same gender, age.

\section{Recommendation}

- Extensive research is also warranted to use standardized parameters.

- $\quad$ Outcomes may be considerable improved in future study if it is done by three dimensional videography.

- Greater scrutiny can be done with large population.

- Further reflection can be done with EMG laboratory.

\section{Ethical Clearance}

As the study includes human subject's ethical clearance is obtained from research and ethical committee of Goutham college of physiotherapy, Bangalore as per the ethical guidelines for Bio-medical research on human subjects, 2000 ICMR, New Delhi.

\section{Bibliography}

1. Brion Conway., et al. "Anterior knee pain in female athletes". Texas Health Ben Hogan Sports Medicine 1 (2013).

2. Boling M., et al. "A Gender differences in the incidence and prevalence of patellofemoral pain syndrome". Scandinavian Journal of Medicine and Science in Sports 20 (2010): 725-730.
3. Joe Heiler Pt CSCS. "Effect of limiting ankle dorsiflexion range of motion on lower extremity kinematics and muscle activation pattern during a squat". Journal of Sport Rehabilitation 21.2 (2012): 144-150.

4. Sara R Piva., et al. "Strength around the hip and flexibility of soft tissues in individuals with and without patellofemoral pain syndrome". Journal of Orthopedic and Sports Physical Therapy 35.12 (2005): 793-801.

5. Mizuno Y., et al. "Quadriceps angle influences tibiofemoral and patellofemoral kinematics". Journal of Orthopaedic Research 19 (2001): 834-840.

6. Edith M Heintjes., et al. "Exercise therapy for patellofemoral pain syndrome". Cochrane Database of Systematic Reviews 4 (2003).

7. Lankhorst NE., et al. "Factors associated with patellofemoral pain syndrome a systematic review". British Journal of Sports Medicine 47.4 (2013): 193-203.

8. Dudzinki K., et al. "The effect of limitation in ankle dorsiflexion on knee joint function: a pilot study". Ortopedia, Traumatologia, Rehabilitacja 15.2 (2013): 44.

9. Thiago Yukio Fukuda., et al. "Hip posterolateral musculature strengthening in sedentary women with patellofemoral pain syndrome. A randomized controlled clinical trial with 1-year follow-up". Journal of Orthopedic and Sports Physical Therapy 2.10 (2012): 823.

10. Cashman GE. "The effect of weak hip abductors or external rotators on knee valgus kinematics in healthy subjects: a systematic 11 review". Journal of Sport Rehabilitation 21.3 (2012): 273-284.

11. Define Kaya., et al. "The effect of an exercise program in conjunction with short-period patellar taping on pain, electromyogram activity, and muscle strength in patellofemoral pain syndrome". American Orthopedic Society for Sports Medicine 2 (2011): 410.

12. Fukuda TY., et al. "Short-term effects of hip abductors and lateral rotators strengthening in females with patellofemoral pain syndrome: a randomized con-trolled clinical trial". Journal of Orthopaedic and Sports Physical Therapy 40 (2010): 736-742. 
13. Bert M Chesworth., et al. "Validation of outcome measures in patients with patellofemoral syndrome". Journal of Orthopaedic and Sports Physical Therapy 10.8 (1989): 30.

14. Michelle C Boling., et al. "Concentric and eccentric torque of the hip musculature in individuals with and without patellofemoral pain". Journal of Athletic Training 44.1 (2009): 7-13.

15. Michael P Reiman., et al. "Hip function's influence on knee dysfunction: A proximal link to a distal problem". Journal of Sport Rehabilitation 18 (2009): 33-46.

16. Theresa Helissa Nakagawa., et al. "The effect of additional strengthening of hip abductor and lateral rotator muscles in patellofemoral pain syndrome: a randomized controlled pilot study". Clinical Rehabilitation 22 (2008): 1051-1060.

17. Timothy F Tyler., et al. "The role of hip muscle function in the treatment of patellofemoral pain syndrome, thirty-five patients with patellofemoral pain syndrome". The American Journal of Sports Medicine 34.4 (2006): 630-633.

18. De Haven KE and Lintner DM. "Athletic injuries: comparison by age, sport, and gender". The American Journal of Sports Medicine 14 (1986): 218-224.

19. Walsh WM., et al. "Recurrent dislocation of the knee in the adult". In: Orthopedic Sports Medicine: Principles and Practice. $2^{\text {nd }}$ ed. Philadelphia, Pa. Saunders (2003): 1718-1721.

20. Carolyn Kisner PT MS., et al. "Therapeutic exercise foundation and techniques". $5^{\text {th }}$ edition (2007).

\section{Assets from publication with us}

- Prompt Acknowledgement after receiving the article

- Thorough Double blinded peer review

- Rapid Publication

- Issue of Publication Certificate

- High visibility of your Published work

Website: www.actascientific.com/

Submit Article: www.actascientific.com/submission.php

Email us: editor@actascientific.com

Contact us: +919182824667

Citation: Shweta Suryvanshi and Nikhil Kumar Rana. "Effect of Knee Extensors Strengthening Versus Hip Posterolateral Musculature Strengthening with Ankle Dorsi Flexors in Patellofemoral Pain". Acta Scientific Orthopaedics 5.3 (2021): 49-56. 